\title{
SYNTHESIS, ANTIMICROBIAL AND $\alpha$-GLUCOSIDASE INHIBITORY POTENTIAL OF MANNICH BASES OF MERCAPTO OXADIAZOLES AND THEIR MOLECULAR DOCKING STUDIES
}

\author{
SAKINA AHMAD ${ }^{1}$, HUMAIRA NADEEM $^{1 *}$, SYED AUN MUHAMMAD $^{2}$, SHAGUFTA NAZ $^{1}$, \\ MUHAMMAD IMRAN ${ }^{1}$, ADIL SAEED ${ }^{1}$ \\ ${ }^{1}$ Department of Pharmaceutical Chemistry, Riphah Institute of Pharmaceutical Sciences, Riphah International University, \\ Islamabad, $7^{\text {th }}$ Avenue, sector G-7/4, 44000, Islamabad, Pakistan \\ ${ }^{2}$ Department of Biotechnology, Bahauddin Zakariya University (BZU), Multan, Pakistan
}

*corresponding author: humaira.nadeem@riphah.edu.pk

Manuscript received: September 2017

\begin{abstract}
In the current study a series of eight Mannich bases were synthesized by incorporating 5-subsituted-1,3,4-oxadiazole-2-thione scaffold. The target compounds were evaluated for their antimicrobial and $\alpha$-glucosidase inhibitory activity. All the synthesized Mannich bases exhibited $\alpha$-glucosidase inhibitory activity with $\mathrm{IC}_{50}$ values between $43.05 \pm 0.11$ and $78.27 \pm$ 0.44 when compared with standard acarbose $27.10 \pm 0.51$. Two compounds $3 \mathbf{c}$ and $\mathbf{4 c}$ containing morpholine moiety exhibited $85 \%$ \& $82 \%$ inhibition as compared to the standard drug (acarbose) which gives $94 \%$ inhibition at dose level of 100 $\mu \mathrm{g} / \mathrm{mL}$. None of the compounds showed significant antimicrobial activity. The interactions of the all compounds with $\alpha-$ glucosidase enzyme were further studied with the help of docking analysis.
\end{abstract}

\section{Rezumat}

În prezentul studiu, a fost sintetizată o serie formată din 8 baze Mannich, prin încorporarea 1,3,4-oxadiazol-2-tionei 5substituite. Compușii țintă au fost evaluați privind acțiunea antimicrobiană și activitatea de inhibare a acestei enzime. Toate bazele Mannich sintetizate au prezentat activitate asupra $\alpha$-glucozidazei, cu valori ale IC $_{50}$ cuprinse între $43,05 \pm 0,11$ și $78,27 \pm 0,44$, comparativ cu valorile de referință ale acarbozei, 27,10 $\pm 0,51$. Doi compuși, 3c și 4c, conținând un rest de morfolină, au prezentat o inhibiţie de $85 \%$ şi respectiv $82 \%$, în comparație cu $94 \%$ pentru substanțe de referinţă, acarboza, la doza de $100 \mu \mathrm{g} / \mathrm{mL}$. Nici unul dintre compuși nu a demonstrat activitate antimicrobiană semnificativă. Interacțiunile tuturor compușilor cu $\alpha$-glucozidaza au fost studiate suplimentar prin analiza de andocare moleculară.

Keywords: synthesis, Mannich bases, $\alpha$-glucosidase inhibitors, diabetes mellitus

\section{Introduction}

Diabetes mellitus is one of the most common endocrine disorder diseases characterized by the hyperglycaemia (defect in insulin secretion, insulin action or both). Diabetes is the major cause of death due to increased cardiovascular risks. Diabetes causes amputation, kidney failure and blindness. The defects in insulin producing beta cells cause postprandial hyperglycaemia, these defects in beta cells plays important role in both types of diabetes progression [12]. The complications caused by hyperglycaemia in diabetic patients include neurological, ophthalmological and renal complications. Diabetes may also lead to atherosclerosis development and cardiac abnormalities. Therefore, strict control of blood glucose concentration is of prime importance to avoid diabetes related complications. The oral glucose absorption can be compromised by targeting $\alpha$ glucosidase enzyme, responsible for carbohydrate digestion [8]. Normally carbohydrates after digestion are converted into simple sugars and absorbed through intestine. Alpha glucosidase inhibitors compete with the $\alpha$ - glucosidase enzyme for the digestion of carbohydrates resulting in less availability of glucose for the body, less postprandial hyperglycaemia and ultimately less insulin secretion. Three $\alpha$-glucosidase inhibitors are available in the market namely acarbose, voglibose and miglitol. These inhibitors are associated with diarrhoea and other GIT problems [22, 29]. Due to the vital role of this enzyme in hyperglycaemia and the side effects of the existing drugs, there is an urgent need to discover safe and effective inhibitors of this key enzyme for the control of diabetic disorders. All nitrogen and sulphur containing heterocyclic rings possess a number of pharmacological activities, especially 1,3,4-oxadiazoles attracted the organic and pharmaceutical chemists to synthesize new drugs in this capacity. 2-Mercato-1,3,4-oxadiazole is another important nucleus which shows broad and effective biological activities like antipsychotic [4], antifungal [7], antiretroviral [11], anti-inflammatory [14], antibacterial [20], anti-parasitic [19], phosphodiesterase and urease's inhibitory inhibition activity $[17,18,24]$. 
FARMACIA, 2018, Vol. 66, 4

Currently, Mannich bases comprising the 1,3,4-oxadiazole ring have gained attention because of their special biological properties. Mannich product is a $\beta$-amino-carbonyl compound, in which primary and secondary amines are used to activate formaldehyde. In this nucleophilic addition an amine is added to carbonyl group and then its hydration leads to Schiff base formation [10]. Mannich bases have gained importance due to their antimicrobial activity [13]. Antibacterial and antifungal activities of norfloxacin Mannich base have been reported [21]. Moreover, heterocyclic compounds Mannich bases possess potent antimicrobial activity $[1,5]$. Other biological activities shown by Mannich bases are antiviral, anti-nociceptive, anti-inflammatory, antiprotozoal and anti-tubercular [30]. Mannich bases comprising of 1,3,4-oxadiazoles ring also showed enormous biological activities like anti-mycobacterial and anti-amoebic $[3,26]$. The antiamoebic activity of 1,3,4-oxadiazole was expected due to the piperazine moiety [28]. In view of the vital role of Mercapto oxadiazoles and Mannich bases in drug discovery, we synthesized some Mannich bases of 2-Mercapto-1,3,4-oxadiazole and evaluated their $\alpha$-glucosidase inhibitory potential.

\section{Materials and Methods}

\section{Chemistry}

All the starting materials, reagents and solvent were purchased from Aldrich, Daejung and Alfa-Aesar. The melting points of the final compounds were also recorded on digital Gallenkamp [SANYO] model MPD.BM 3.5 apparatus and were uncorrected. ${ }^{1} \mathrm{H}-$ NMR and ${ }^{13} \mathrm{C}$-NMR spectra were recorded on Bruker AM-300 in DMSO-d $\mathrm{d}_{6}$ at $300 \mathrm{MHz}$ and $75 \mathrm{MHz}$ respectively, using TMS as an internal standard. Alpha Bruker FTIR spectrophotometer (ATR eco ZnSe, $v_{\max }$ in $\mathrm{cm}^{-1}$ ) was used to record FTIR spectra. Elemental analysis was carried out using LECO-183 CHNS analyser. All reactions were monitored by thin layer chromatography (TLC) using solvent system (ethyl acetate:petroleum ether $\mathrm{v} / \mathrm{v} 3: 1$ ).

All the target compounds were synthesized according to Figure 1.

General procedure for the synthesis of hydrazides (1a, 1b)

Hydrazine hydrate $(0.03 \mathrm{~mol})$ was added to the already stirred solution of methyl salicylate/methyl anthranilate $(0.01 \mathrm{~mol})$ in $30 \mathrm{~mL}$ ethanol. The reaction mixture was refluxed for 5 - $6 \mathrm{hrs}$ and cooled overnight. The excess solvent and hydrazine was removed using rotary evaporator. The resulting solid product was filtered, recrystallized from ethanol and dried.

2-Aminobenzhydrazide (1a)

Yield: 48\%; m.p. (obs.) $118-120^{\circ} \mathrm{C}$; m.p. (lit.) [32] $121-125^{\circ} \mathrm{C}, \mathrm{R}_{\mathrm{f}}: 0.34$ (ethyl acetate:petroleum ether $\mathrm{v} / \mathrm{v} 3: 1)$. 2-hydroxybenzhydrazide (1b)

Yield: 77\%; m.p. (obs.) $144-146^{\circ} \mathrm{C}$; m.p. (lit.) [23] $147-150^{\circ} \mathrm{C}, \mathrm{R}_{\mathrm{f}}: 0.49$ (ethyl acetate:petroleum ether v/v $3: 1)$.
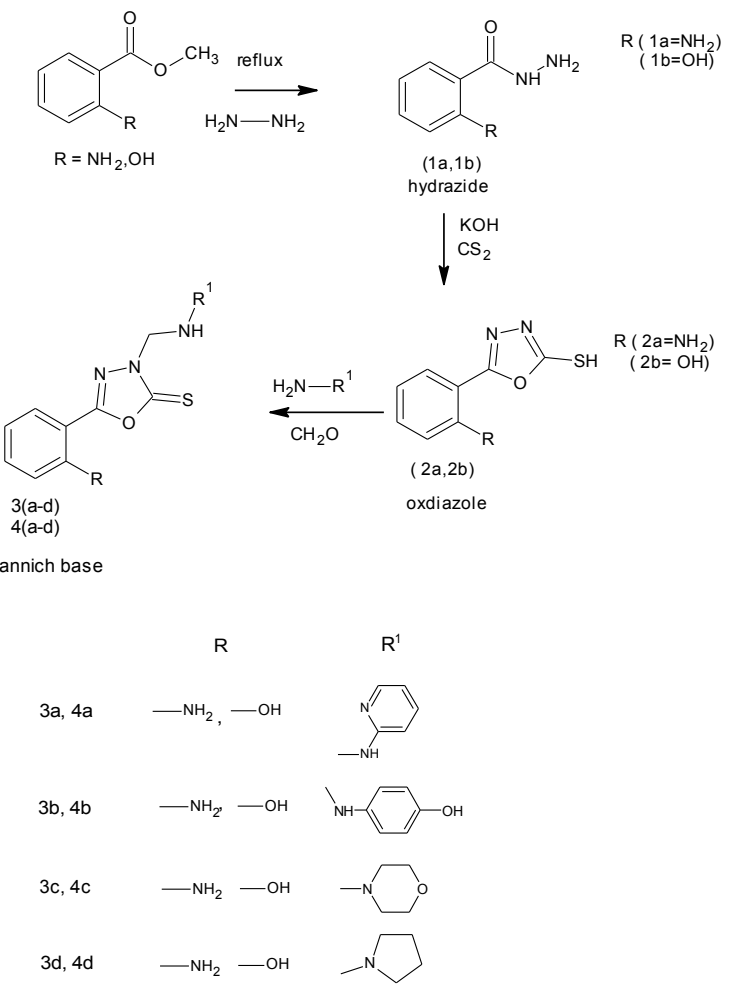

Figure 1.

Synthesis of Mannich bases of 5-subsituted-1,3,4oxadiazole-2-thione

General procedure for the synthesis of 1,3,4-oxadiazole-2-thione $(\mathbf{2 a}, \mathbf{2 b})$

To the solution of hydrazide $(0.025 \mathrm{~mol})$ in absolute ethanol, $\mathrm{CS}_{2}(0.030 \mathrm{~mol})$ was added followed by the addition of $0.025 \mathrm{~mol}$ of $\mathrm{KOH}$ dissolved in $30 \mathrm{~mL}$ of distilled water. The above reaction mixture was heated and stirred under reflux till the evolution of $\mathrm{H}_{2} \mathrm{~S}$ ceased. After completion of reaction the reaction mixture was concentrated in vacuo. Distilled water was added to the residue and the solution was acidified with dilute $\mathrm{HCl}$ to $\mathrm{pH}$ of $2-3$. The precipitates were filtered, washed with diethyl ether and recrystallized from ethanol [15].

5-(2-aminophenyl)-1,3,4-oxadiazole-2(3H)-thione

(2a)

Yield: 52\%; m.p. (obs.) $152-154^{\circ} \mathrm{C}$, m.p. (lit.) [25]

$155-157^{\circ} \mathrm{C}, \mathrm{R}_{\mathrm{f}}$ : 0.72 (ethyl acetate:petroleum ether v/v 3:1); IR $\left(\mathrm{cm}^{-1}\right): 3367(\mathrm{NH}), 1624(\mathrm{C}=\mathrm{C}), 1504$ $(\mathrm{C}=\mathrm{N})$; ${ }^{1} \mathrm{H}-\mathrm{NMR}\left(300 \mathrm{MHz}, \mathrm{DMSO}-\mathrm{d}_{6}\right): \delta 6.65-$ 7.20 (m, 8H, Ar-H), 3.35 (d, 2H, $\mathrm{NH}_{2}$ ), 1.6 ppm (s, $1 \mathrm{H}, \mathrm{SH})$.

5-(2-hydroxyphenyl)-1,3,4-oxadiazole-2(3H)-thione (2b)

Yield: $68 \%$; m.p. (obs.) $162-163^{\circ} \mathrm{C}$, m.p (lit.) [9] $162-164^{\circ} \mathrm{C}$; $\mathrm{R}_{\mathrm{f}}$ : 0.75 (ethyl acetate:petroleum ether 
FARMACIA, 2018, Vol. 66, 4

v/v 3:1); IR $\left(\mathrm{cm}^{-1}\right): 3188(\mathrm{OH}), 1611(\mathrm{C}=\mathrm{C}), 1495$ $(\mathrm{C}=\mathrm{N}) ;{ }^{1} \mathrm{H}-\mathrm{NMR}\left(300 \mathrm{MHz}, \mathrm{DMSO}-\mathrm{d}_{6}\right): \delta 10.46(\mathrm{~s}$, $1 \mathrm{H}, \mathrm{OH}), 6.43$ - 7.28 (m, 8H, Ar-H), 1.89 ppm (s, $1 \mathrm{H}, \mathrm{SH})$.

General procedure for the synthesis of 5-subsituted1,3,4-oxadiazole-2-thione Mannich bases (3(a-d), $4(a-d))$

Oxadiazole-2-thiones $\mathbf{2 a}, \mathbf{2 b}(0.01 \mathrm{~mol})$ and different amines $(0.01 \mathrm{~mol})$ were mixed in $30 \mathrm{~mL}$ of ethanol and stirred for 15 minutes. To the stirred suspension formaldehyde $(0.05 \mathrm{~mol})$ was added drop wise and heated to reflux for $5 \mathrm{hrs}$ with stirring. The progress of reaction was monitored by TLC. The crude product was separated as solid on cooling after the completion of reaction. The product was filtered and recrystallized from ethanol [27].

5-(2-aminophenyl)-3-[(pyridin-2-ylamino)methyl]1,3,4-oxadiazole-2(3H)-thione (3a)

Yield: $56 \%$; m.p. $300^{\circ} \mathrm{C}$ (decomp.), $\mathrm{R}_{\mathrm{f}}$ : 0.15 (ethyl acetate:petroleum ether $\mathrm{v} / \mathrm{v} 3: 1)$; IR $\left(\mathrm{cm}^{-1}\right) 3367(\mathrm{NH})$, $2916(\mathrm{CH}), 1635(\mathrm{C}=\mathrm{C}), 1562(\mathrm{C}=\mathrm{N}), 1237 \mathrm{~cm}^{-1}$ $(\mathrm{C}=\mathrm{S}) ;{ }^{1} \mathrm{H}-\mathrm{NMR}\left(300 \mathrm{MHz}, \mathrm{DMSO}-\mathrm{d}_{6}\right): \delta 6.58-$ $7.36(\mathrm{~m}, 4 \mathrm{H}, \mathrm{Ar}-\mathrm{H}), 6.02-6.48(\mathrm{~m}, 5 \mathrm{H}$, pyridyl-H), $4.81\left(\mathrm{~s}, 2 \mathrm{H}, \mathrm{CH}_{2}\right), 3.23 \mathrm{ppm}\left(\mathrm{s}, 2 \mathrm{H}, \mathrm{NH}_{2}\right),{ }^{13} \mathrm{C}-$ NMR (75 MHz, DMSO-d 6 ): $\delta 176.3,157.9,153.2$, 150.4, 146.5, 139.7, 133.6, 129.6, 121.4, 117.3, 116.2, 113.5, 110.6, 59.7. Elemental analysis: $\mathrm{C}_{14} \mathrm{H}_{13} \mathrm{~N}_{5} \mathrm{OS}$; Calculated: C 56.17, N 23.40, H 4.38\%; Found: C $56.21, \mathrm{~N} 23.25, \mathrm{H} 4.35 \%$.

5-(2-aminophenyl)-3-\{[(4-hydroxyphenyl)amino]methyl\}-1,3,4-oxadiazole-2(3H)-thione (3b)

Yield: $55 \%$; m.p. $180-182^{\circ} \mathrm{C}$; $\mathrm{R}_{\mathrm{f}}$ : 0.33 (ethyl acetate: petroleum ether v/v 3:1); IR $\left(\mathrm{cm}^{-1}\right) 3055(\mathrm{NH}), 2955$ $(\mathrm{CH}), 1609(\mathrm{C}=\mathrm{C}), 1219 \mathrm{~cm}^{-1}(\mathrm{C}=\mathrm{S}) ;{ }^{1} \mathrm{H}-\mathrm{NMR}(300$ MHz, DMSO-d ${ }_{6}$ ): $\delta 10.9$ (s, IH, OH), 6.23 - 7.42 (m, 4H, Ar-H), 5.41 (s, 2H, $\left.\mathrm{CH}_{2}\right), 3.6 \mathrm{ppm}(\mathrm{s}, 2 \mathrm{H}$, $\left.\mathrm{NH}_{2}\right) ;{ }^{13} \mathrm{C}-\mathrm{NMR}\left(75 \mathrm{MHz}, \mathrm{DMSO}-\mathrm{d}_{6}\right): \delta 174.8,157.5$, 155.2, 148.3, 140.5, 134.2, 129.8, 121.5, 120.2, 117.6, 116.5, 114.9, 112.5, 110.4, 56.8. Elemental analysis: $\mathrm{C}_{15} \mathrm{H}_{14} \mathrm{~N}_{4} \mathrm{O}_{2} \mathrm{~S}$; Calculated: C 57.31, N 17.82, H 4.49\%; Found: C 57.43, N 17.29, H 4.36\%.

5-(2-aminophenyl)-3-(morpholin-4-ylmethyl)-1,3,4oxadiazole-2(3H)-thione (3c)

Yield: $60 \%$; m.p. $230-235^{\circ} \mathrm{C}$; $\mathrm{R}_{\mathrm{f}}: 0.35$ (ethyl acetate: petroleum ether v/v 3:1); IR $\left(\mathrm{cm}^{-1}\right) 3216(\mathrm{NH}), 2919$ $(\mathrm{CH}), 1457(\mathrm{C}=\mathrm{N}), 1267(\mathrm{C}=\mathrm{C}), 1266 \mathrm{~cm}^{-1}(\mathrm{C}=\mathrm{S})$, ${ }^{1} \mathrm{H}-\mathrm{NMR}\left(300 \mathrm{MHz}, \mathrm{DMSO}-\mathrm{d}_{6}\right): \delta 6.01-7.74(\mathrm{~m}$, $4 \mathrm{H}, \mathrm{Ar}-\mathrm{H}), 4.96$ (s, 2H, $\left.\mathrm{CH}_{2}\right), 3.72-3.81(\mathrm{~m}, 8 \mathrm{H}$, morpholine-H), $3.53 \mathrm{ppm}\left(\mathrm{s}, 2 \mathrm{H}, \mathrm{NH}_{2}\right) .{ }^{13} \mathrm{C}-\mathrm{NMR}$ $\left(75 \mathrm{MHz}, \mathrm{DMSO}-\mathrm{d}_{6}\right): \delta 177.5,156.6,150.5,135.3$, 129.5, 118.7, 116.3, 111.6, 69.5, 66.2, 62.5, 49.2, 46.7. Elemental analysis: $\mathrm{C}_{13} \mathrm{H}_{16} \mathrm{~N}_{4} \mathrm{O}_{2} \mathrm{~S}$; Calculated: C 53.41, N 19.16, H 5.52\%; Found: C 53.23, N 19.25, H $5.43 \%$.

5-(2-aminophenyl)-3-(pyrrolidin-4-ylmethyl)-1,3,4oxadiazole-2(3H)-thione (3d)

Yield: 67\%; m.p. $245-247^{\circ} \mathrm{C}$; $\mathrm{R}_{\mathrm{f}}$ : 0.37 (ethyl acetate: petroleum ether v/v 3:1); IR ( $\left.\mathrm{cm}^{-1}\right) 3391(\mathrm{NH}), 2921$
$(\mathrm{CH}), 1666(\mathrm{C}=\mathrm{C}), 1463(\mathrm{C}=\mathrm{N}), 1270 \mathrm{~cm}^{-1}(\mathrm{C}=\mathrm{S})$, ${ }^{1} \mathrm{H}-\mathrm{NMR}\left(300 \mathrm{MHz}, \mathrm{DMSO}-\mathrm{d}_{6}\right): \delta 6.65-6.95(\mathrm{~m}$, $4 \mathrm{H}, \mathrm{Ar}-\mathrm{H}), 5.59$ (s, 2H, $\left.\mathrm{CH}_{2}\right), 3.54-3.63(\mathrm{~m}, 8 \mathrm{H}$, pyrrolidine-H), $3.11 \mathrm{ppm}\left(\mathrm{s}, 2 \mathrm{H}, \mathrm{NH}_{2}\right) ;{ }^{13} \mathrm{C}-\mathrm{NMR}$ $\left(75 \mathrm{MHz}, \mathrm{DMSO}-\mathrm{d}_{6}\right): \delta 172.5,160.3,150.2,132.1$, $128.5,117.6,115.5,109.2$, 67.3, 49.5, 45.2, 35.4, 23.7. Elemental analysis: $\mathrm{C}_{13} \mathrm{H}_{16} \mathrm{~N}_{4} \mathrm{OS}$; Calculated: $\mathrm{C}$ 56.50, N 20.27, H 5.84\%; Found: C 56.42, N 20.28, $\mathrm{H} 5.69 \%$.

5-(2-hydroxyphenyl)-3-[(pyridine-2-ylamino)methyl]1,3,4-oxadiazole-2(3H)-thione (4a)

Yield: 54\%; m.p. $205-207^{\circ}$ C; m.p. (lit.) [2] 207 $209^{\circ} \mathrm{C} \mathrm{R}_{\mathrm{f}}: 0.27$ (ethyl acetate:petroleum ether $\mathrm{v} / \mathrm{v}$ 3:1), IR( $\left.\mathrm{cm}^{-1}\right) 3353(\mathrm{OH}), 2935(\mathrm{CH}), 1610(\mathrm{C}=\mathrm{C})$, $1517(\mathrm{C}=\mathrm{N}), 1271 \mathrm{~cm}^{-1}(\mathrm{C}=\mathrm{S}) ;{ }^{1} \mathrm{H}-\mathrm{NMR}(300 \mathrm{MHz}$, DMSO-d $\left.{ }_{6}\right): \delta 10.44$ (s, IH, OH), $8.03(\mathrm{dd}, J=5.0$, $7.1 \mathrm{~Hz}, 1 \mathrm{H}$, pyridyl-H), 7.71 (dt, $J=7.2,1.2 \mathrm{~Hz}, 1 \mathrm{H}$, pyridyl-H), 7.49 (dt, $J=10.0,2.5 \mathrm{~Hz}, 1 \mathrm{H}$, pyridylH), 7.43 (dt, $J=10.5,3.0 \mathrm{~Hz}, 1 \mathrm{H}$, pyridyl-H), 6.62 $7.02(\mathrm{~m}, 4 \mathrm{H}, \mathrm{Ar}-\mathrm{H}), 5.64\left(\mathrm{~s}, 3 \mathrm{H}, \mathrm{CH}_{2}\right) .{ }^{13} \mathrm{C}-\mathrm{NMR}$ $\left(75 \mathrm{MHz}, \mathrm{DMSO}-\mathrm{d}_{6}\right): \delta 175.2,159.5,156.7,151.2$, 148.5, 139.4, 131.8, 127.5, 120.6, 118.7, 116.5, 111.5, 102.4, 55.5. Elemental analysis: $\mathrm{C}_{14} \mathrm{H}_{12} \mathrm{~N}_{4} \mathrm{O}_{2} \mathrm{~S}$; Calculated: C 55.99, N 18.65, H 4.03\%; Found: C 55.56, N 18.54, H 4.15\%.

5-(2-hydroxyphenyl)-3-\{[(4-hydroxyphenyl) amino] methyl\}-1,3,4-oxadiazole-2(3H)-thione (4b)

Yield: $80 \%$; m.p. $195-197^{\circ} \mathrm{C}$; $\mathrm{R}_{\mathrm{f}}$ : 0.12 (ethyl acetate: petroleum ether v/v 3:1); IR $\left(\mathrm{cm}^{-1}\right) 3425(\mathrm{OH}), 2980$ $(\mathrm{CH}), 1650(\mathrm{C}=\mathrm{C}), 1490(\mathrm{C}=\mathrm{N}), 1237 \mathrm{~cm}^{-1}(\mathrm{C}=\mathrm{S})$; ${ }^{1} \mathrm{H}-\mathrm{NMR}\left(300 \mathrm{MHz}, \mathrm{DMSO}-\mathrm{d}_{6}\right): \delta 10.91(\mathrm{~s}, 1 \mathrm{H}, \mathrm{OH})$, 9.78 (s, 1H, OH), 6.78 - 7.86 (m, 4H, Ar-H), 4.58 ppm $\left(\mathrm{s}, 2 \mathrm{H}, \mathrm{CH}_{2}\right) ;{ }^{13} \mathrm{C}-\mathrm{NMR}\left(75 \mathrm{MHz}, \mathrm{DMSO}-\mathrm{d}_{6}\right): \delta$ $179.8,162.5,155.5,150.2,143.5,132.7,127.4,122.8$, $120.5,119.3,117.5,114.2,112.3,110.6,54.5$. Elemental analysis: $\mathrm{C}_{15} \mathrm{H}_{13} \mathrm{~N}_{3} \mathrm{O}_{3} \mathrm{~S}$; Calculated: $\mathrm{C}$ 57.13, N 13.33, H 4.16\%; Found: C 57.10, N 13.15, H 4.05\%. 5-(2-hydroxyphenyl)-3-(morpholin-4-ylmethyl)1,3,4-oxadiazole-2(3H)-thione $(4 \mathrm{c})$

Yield: $70 \%$; m.p. $185-187^{\circ} \mathrm{C}$; m.p. (lit.) [27] 184 $186^{\circ} \mathrm{C}, \mathrm{R}_{\mathrm{f}}: 0.18$ (ethyl acetate:petroleum ether $\mathrm{v} / \mathrm{v}$ 3:1); IR ( $\left.\mathrm{cm}^{-1}\right) 3470(\mathrm{OH}), 2942(\mathrm{CH}), 1675(\mathrm{C}=\mathrm{C})$, $1485(\mathrm{C}=\mathrm{N}), 1190 \mathrm{~cm}^{-1}(\mathrm{C}=\mathrm{S}) ;{ }^{1} \mathrm{H}-\mathrm{NMR}(300 \mathrm{MHz}$, DMSO-d $\left.{ }_{6}\right): \delta 11.80(\mathrm{~s}, \mathrm{IH}, \mathrm{OH}), 6.96-7.58(\mathrm{~m}, 4 \mathrm{H}$, Ar-H), 5.38 (s, 2H, $\left.\mathrm{CH}_{2}\right), 3.48-3.72(\mathrm{~m}, 8 \mathrm{H}$, morpholine-H); ${ }^{13} \mathrm{C}-\mathrm{NMR}\left(75 \mathrm{MHz}, \mathrm{DMSO}_{-}\right.$): $\delta$ 175.2, 160.5, 155.4, 135.3, 130.5, 119.4, 118.2, 112.7, 70.5, 67.6, 64.5, 53.2, 50.6. Elemental analysis: $\mathrm{C}_{13} \mathrm{H}_{15} \mathrm{~N}_{3} \mathrm{O}_{3} \mathrm{~S}$; Calculated: C 53.23, N 14.32, H 5.15\%; Found: C 53.15, N 14.45, H 5.08\%.

5-(2-hydroxyphenyl)-3-(pyrrolidin-4-ylmethyl)-1,3,4oxadiazole-2(3H)-thione (4d)

Yield: $60 \%$; m.p. $230-233^{\circ} \mathrm{C}$; $\mathrm{R}_{\mathrm{f}}$ : 0.32 (ethyl acetate: petroleum ether v/v 3:1); IR $\left(\mathrm{cm}^{-1}\right) 3328(\mathrm{OH}), 2930$ $(\mathrm{CH}), 1618(\mathrm{C}=\mathrm{C}), 1452(\mathrm{C}=\mathrm{N}), 1253 \mathrm{~cm}^{-1}(\mathrm{C}=\mathrm{S})$; ${ }^{1} \mathrm{H}-\mathrm{NMR}\left(300 \mathrm{MHz}, \mathrm{DMSO}-\mathrm{d}_{6}\right): \delta 10.75(\mathrm{~s}, 1 \mathrm{H}, \mathrm{OH})$, 7.05 - 7.32 (m, 4H, Ar-H), 5.29 (s, 2H, $\left.\mathrm{CH}_{2}\right), 2.50$ $3.68\left(\mathrm{~m}, 8 \mathrm{H}\right.$, pyrrolidine-H); ${ }^{13} \mathrm{C}-\mathrm{NMR}(300 \mathrm{MHz}$, 
FARMACIA, 2018, Vol. 66, 4

DMSO-d $\left.{ }_{6}\right): \delta 175.7,160.4,155.5,131.4,129.8$, 120.2, 118.8, 114.5, 63.2, 52.5, 51.1, 26.5, 22.4. Elemental analysis: $\mathrm{C}_{13} \mathrm{H}_{15} \mathrm{~N}_{3} \mathrm{O}_{2} \mathrm{~S}$; Calculated: $\mathrm{C} 56.30$, N 15.15, H 5.45\%; Found: C 56.43, N 15.24, H 5.36\%. Antimicrobial activities assay

The antibacterial and antifungal activities of the synthesized Mannich bases 3(a-d) and 4(a-d) were evaluated by disc diffusion method [6, 16]. Three bacterial strains namely Escherichia coli (ATCC25922), Pseudomonas aeruginosa (ATCC 10145), Staphylococcus aureus (ATCC 6538) and two fungal strains, Candida glabrata (ATCC 62934) and Candida albicans (ATCC 60387) were used. Gentamycin sulphate was taken as positive control for the antibacterial activity and nystatin was taken as positive control for the antifungal activity. DMSO was taken as negative control. Nutrient agar/Sabouraud dextrose agar and nutrient broth were sterilized in the autoclave at $121^{\circ} \mathrm{C}$ for 15 minutes. Sterile agar plates were prepared by pouring $25 \mathrm{~mL}$ sterile nutrient agar into each plate and kept into the incubator at $37^{\circ} \mathrm{C}$ for 24 hours. The fresh bacterial/fungal strains were prepared in sterile nutrient broth and incubated at $37^{\circ} \mathrm{C}$ for $24 \mathrm{hrs}$. The discs of Whatmann filter paper were prepared and sterilized in autoclave. Lawn

$$
\% \alpha \text {-glucosidase inhibition }=\left(\mathrm{Abs}_{\text {blank }}-\mathrm{Abs}_{\text {test comp }}\right) / \mathrm{Abs}_{\text {blank }} * 100 .
$$

All the compounds were suitably diluted and their inhibition studies were determined. Results were obtained as \% inhibition and $\mathrm{IC}_{50}$ values were calculated graphically by plotting log inhibitor concentration versus percent inhibition.

\section{Molecular Docking studies}

Preparation of ligand molecules. The chemical structures of 3(a-d) and 4(a-d) were prepared by ChemBio Draw and MOL format of these ligands were generated.

Accession of targets proteins. The 3D crystal structure of alpha-glucosidase (PDB ID: 3WY1) was accessed and downloaded from Protein Data Bank (PDB) database.

Analysis of target active binding sites. The active sites of the targeted protein were analysed using the Molecular Operating Environment (MOE) software. An active site was defined from the coordinates of the ligand in the original target protein site.

Docking. Computational ligand-target docking approach was used to determine structural complexes of the crystal structure of alpha-glucosidase protein target with ligand molecules in order to understand the structural basis of the protein target specificity. Finally, docking was carried out by Molecular Operating Environment (MOE) software. The energy of interaction of these derivatives with the protein targets was assigned "grid point". of each bacterial/fungal strain was prepared and different concentrations $(3 \mu \mathrm{g}, 10 \mu \mathrm{g}, 30 \mu \mathrm{g}, 100 \mu \mathrm{g}$, $300 \mu \mathrm{g}, 700 \mu \mathrm{g})$ of test compound were placed on each agar plate along with standard reference disc. The plates were incubated at $37^{\circ} \mathrm{C}$ for 24 hours in case of antibacterial assay and at $28^{\circ} \mathrm{C}$ for four days in the antifungal screening assay. The zone of inhibitions was measured accordingly.

$\alpha$-Glucosidase enzyme inhibition assay

In vitro $\alpha$-glucosidase inhibitory activity was performed by following reported procedure [31]. $100 \mu \mathrm{L}$ of reaction mixture was prepared using $70 \mu \mathrm{L}$ of $50 \mathrm{mM}$ phosphate buffer $\mathrm{pH}=6.8,10 \mu \mathrm{L}$ of test compound followed by the addition of $10 \mu \mathrm{L}$ (0.057 units) enzyme solution in the buffer. The contents were mixed, pre-incubated for 10 minutes at $25^{\circ} \mathrm{C}$ and absorbance measured at $400 \mathrm{~nm}$. The reaction was initiated by the addition of $10 \mu \mathrm{L}$ of $0.5 \mathrm{mM} p$-nitrophenylglucopyranoside and incubated at $25^{\circ} \mathrm{C}$, the absorbance of $p$-nitrophenol was measured at $400 \mathrm{~nm}$ using the 96-well plate reader. Acarbose was used as standard. All the experiments were carried out in triplicates. The percentage inhibition was calculated by the given formula:

\section{Results and Discussion}

\section{Chemistry}

All the Mannich bases were synthesized according to the Figure 1. In the first step, hydrazides (1a, 1b) were synthesized by the reaction of the respective esters (methyl salicylate, methyl anthranilate) with the hydrazine hydrate, using absolute ethanol as solvent. These hydrazides were further condensed with carbon disulphide and potassium hydroxide to get the corresponding 5-subsituted-1,3,4-oxadiazoles-2-thiones $(\mathbf{2 a}, \mathbf{2 b})$ following the reported procedure. Finally, the synthesized 5-subsituted-1,3,4-oxadiazole-2-thione were treated with different primary and secondary amines (2-aminopyridine, 4-hydroxyphenol, morpholine, pyrrolidine) and formaldehyde to furnish the target Mannich bases 3(a-d) and 4(a-d). The structures of all the Mannich base derivatives of oxadiazoles were characterized by IR, ${ }^{1} \mathrm{H}-\mathrm{NMR}$ and ${ }^{13} \mathrm{C}-\mathrm{NMR}$ spectroscopic data.

IR spectra of compounds 3(a-d) and 4(a-d) showed moderate intensity absorption bands between 3055 $3470 \mathrm{~cm}^{-1}$ for $\mathrm{NH}$ stretching vibrations. Strong bands of $\mathrm{C}=\mathrm{N}$ and $\mathrm{C}=\mathrm{S}$ were observed at $1452-1562 \mathrm{~cm}^{-1}$ and $1190-1271 \mathrm{~cm}^{-1}$ respectively thus indicating the formation of Mannich bases. In the ${ }^{1} \mathrm{H}-\mathrm{NMR}$ spectra of compounds, the signals of $-\mathrm{CH}_{2}-$ protons appeared in the range $4.81-5.64 \mathrm{ppm}$ in the downfield region due to the peri effect of two nitrogen atoms thus confirming the formation of mannich bases. In case of compounds $\mathbf{3 ( a - d )}, \mathrm{NH}_{2}$ protons 
FARMACIA, 2018, Vol. 66, 4

resonated as singlet at $3.11-3.60 \mathrm{ppm}$ whereas in 4(a-d) singlet of $\mathrm{OH}$ proton appeared downfield at $10.44-11.80 \mathrm{ppm}$. The protons of pyridyl group in case of compound $\mathbf{3} \mathbf{a}$ and $\mathbf{4 a}$ resonated in the range $7.43-8.03 \mathrm{ppm}$ while morpholine protons of $\mathbf{3 c}$ and $\mathbf{4 c}$ were observed up-field at $3.42-3.81 \mathrm{ppm}$ as multiplets. In case of compounds $\mathbf{3 d}$ and $\mathbf{4 d}$ bearing pyrrolidinyl moiety, the multipletfor pyrrolidine protons appeared at $2.50-3.68 \mathrm{ppm}$.

Antimicrobial activity

The Mannich bases 3(a-d) exhibited low to moderate activity except few compounds which showed no activity (Table I).

Table I

Antimicrobial activity of synthesized Mannich bases 3(a-d) \& 4(a-d)

\begin{tabular}{|c|c|c|c|c|c|}
\hline & ntimicrobial activ & ity of synthesized Mannich & bases and their zone of ir & hibition measured i & $\mathrm{n}(\mathrm{mm})$ \\
\hline \multirow{3}{*}{ Compounds } & \multicolumn{3}{|c|}{ Bacterial strains } & \multicolumn{2}{|c|}{ Fungal strains } \\
\hline & \multicolumn{2}{|r|}{ Gram (-) ive } & \multirow{2}{*}{$\begin{array}{c}\text { Gram }(+) \text { ive } \\
\text { Staphylococcus aureus }\end{array}$} & \multirow[b]{2}{*}{ Candida glabrata } & \multirow[b]{2}{*}{ Candida albicans } \\
\hline & \multicolumn{2}{|c|}{\begin{tabular}{|l|l|} 
Escherichia coli & Pseudomonas aeruginosa
\end{tabular}} & & & \\
\hline $3 \mathbf{a}$ & & & & & \\
\hline $3 \mu \mathrm{g}$ & 5 & 3 & --- & --- & 2 \\
\hline $10 \mu \mathrm{g}$ & 5 & 3 & --- & --- & 3 \\
\hline $30 \mu \mathrm{g}$ & 6 & 4 & 3 & --- & 3 \\
\hline $100 \mu \mathrm{g}$ & 7 & 5 & 3 & 0.5 & 4 \\
\hline $300 \mu \mathrm{g}$ & 10 & 5 & 4 & 1 & 4 \\
\hline $700 \mu \mathrm{g}$ & 10 & 5 & 4 & 2 & 4 \\
\hline $\mathbf{3 b}$ & & & & & \\
\hline $3 \mu \mathrm{g}$ & --- & --- & --- & 2 & --- \\
\hline $10 \mu \mathrm{g}$ & 4 & 3 & 2 & 2 & --- \\
\hline $30 \mu \mathrm{g}$ & 6 & 2 & 2 & 3 & --- \\
\hline $100 \mu \mathrm{g}$ & 10 & 3 & 3 & 4 & 4 \\
\hline $300 \mu \mathrm{g}$ & 11 & 5 & 4 & 5 & 5 \\
\hline $700 \mu \mathrm{g}$ & 14 & 6 & 4 & 5 & 5 \\
\hline $3 \mathbf{c}$ & & & & & \\
\hline $3 \mu \mathrm{g}$ & 3 & 4 & 3 & 1 & 1 \\
\hline $10 \mu \mathrm{g}$ & 4 & 4 & 4 & 2 & 2 \\
\hline $30 \mu \mathrm{g}$ & 7 & 5 & 5 & 2 & 3 \\
\hline $100 \mu \mathrm{g}$ & 7 & 6 & 6 & 3 & 4 \\
\hline $300 \mu \mathrm{g}$ & 10 & 10 & 6 & 4 & 4 \\
\hline $700 \mu \mathrm{g}$ & 12 & 12 & 7 & 5 & 5 \\
\hline $3 d$ & & & & & \\
\hline $3 \mu \mathrm{g}$ & 2 & 4 & --- & --- & --- \\
\hline $10 \mu \mathrm{g}$ & 3 & 5 & --- & 3 & --- \\
\hline $30 \mu \mathrm{g}$ & 3 & 7 & --- & 3 & --- \\
\hline $100 \mu \mathrm{g}$ & 4 & 8 & 3 & 4 & 3 \\
\hline $300 \mu \mathrm{g}$ & 7 & 10 & 4 & 7 & 4 \\
\hline $700 \mu \mathrm{g}$ & 8 & 12 & 5 & 8 & 6 \\
\hline $4 a$ & & & & & \\
\hline $3 \mu \mathrm{g}$ & 2 & --- & 4 & 1 & --- \\
\hline $10 \mu \mathrm{g}$ & 3 & --- & 4 & 2 & --- \\
\hline $30 \mu \mathrm{g}$ & 10 & 5 & 5 & 3 & --- \\
\hline $100 \mu \mathrm{g}$ & 10 & 5 & 6 & 3 & 2 \\
\hline $300 \mu \mathrm{g}$ & 17 & 7 & 7 & 4 & 3 \\
\hline $700 \mu \mathrm{g}$ & 18 & 8 & 9 & 4 & 3 \\
\hline $4 \mathrm{~b}$ & & & & & \\
\hline $3 \mu \mathrm{g}$ & --- & 2 & 2 & --- & 1 \\
\hline $10 \mu \mathrm{g}$ & --- & 4 & 3 & --- & 1 \\
\hline $30 \mu \mathrm{g}$ & 2 & 3 & 7 & 2 & 2 \\
\hline $100 \mu \mathrm{g}$ & 7 & 3 & 8 & 2 & 3 \\
\hline $300 \mu \mathrm{g}$ & 10 & 10 & 6 & 3 & 4 \\
\hline $700 \mu \mathrm{g}$ & 10 & 6 & 5 & 4 & 4 \\
\hline $4 c$ & & & & & \\
\hline $3 \mu \mathrm{g}$ & 3 & 3 & --- & --- & --- \\
\hline $10 \mu \mathrm{g}$ & 3 & 7 & 2 & --- & 2 \\
\hline $30 \mu \mathrm{g}$ & 4 & 8 & 3 & 2 & 3 \\
\hline $100 \mu \mathrm{g}$ & 8 & 9 & 5 & 3 & 5 \\
\hline $300 \mu \mathrm{g}$ & 6 & 9 & 5 & 6 & 3 \\
\hline $700 \mu \mathrm{g}$ & 15 & 10 & 7 & 8 & 6 \\
\hline
\end{tabular}


FARMACIA, 2018, Vol. 66, 4

\begin{tabular}{|c|c|c|c|c|c|}
\hline & ntimicrobial activ & ity of synthesized Mannich & bases and their zone of $\mathrm{i}$ & hibition measured it & $\mathrm{n}(\mathrm{mm})$ \\
\hline \multirow{3}{*}{ Compounds } & \multicolumn{3}{|c|}{ Bacterial strains } & \multicolumn{2}{|c|}{ Fungal strains } \\
\hline & \multicolumn{2}{|r|}{ Gram - } & \multirow{2}{*}{$\begin{array}{c}\text { Gram }+ \\
\text { Staphylococcus aureus }\end{array}$} & \multirow[b]{2}{*}{ Candida glabrata } & \multirow[b]{2}{*}{ Candida albicans } \\
\hline & Escherichia coli & Pseudomonas aeruginosa & & & \\
\hline 4d & & & & & \\
\hline $3 \mu \mathrm{g}$ & 2 & 5 & 2 & --- & 2 \\
\hline $10 \mu \mathrm{g}$ & 2 & 7 & 5 & --- & 5 \\
\hline $30 \mu \mathrm{g}$ & 3 & 8 & 6 & 2 & 4 \\
\hline $100 \mu \mathrm{g}$ & 3 & 9 & 7 & 5 & 7 \\
\hline $300 \mu \mathrm{g}$ & 4 & 6 & 7 & 6 & 7 \\
\hline $700 \mu \mathrm{g}$ & 8 & 12 & 8 & 8 & 10 \\
\hline $\begin{array}{l}\text { Gentamycin } \\
\text { sulphate }\end{array}$ & 24.1 & 23 & 24.3 & --- & --- \\
\hline Nystatin & --- & ---- & ---- & 19 & 19.2 \\
\hline
\end{tabular}

Compound 3b, having the $p$-hydroxy-phenyl moiety showed greater zone of inhibition against Escherichia coli i.e. $14 \mathrm{~mm}$ while compound 3c containing morpholine moiety exhibited moderate antibacterial activity i.e. $12 \mathrm{~mm}$ against both the gram negative strains. Compound 3a and 3d possess low activity against all the strains which indicated that pyridine and pyrolidine moieties do not contribute to a significant antimicrobial activity of Mannich bases. All the Mannich bases 3(a-d) did not show antifungal activity.

The compounds $\mathbf{4 ( a - d )}$ also showed low to moderate antibacterial activity. Compound $\mathbf{4 a}$, having pyridine moiety exhibited moderate antibacterial activity against
Escherichia coli with a zone of inhibition of $18 \mathrm{~mm}$. In case of compound $\mathbf{4 c}$ mild activity was observed against Escherchia coli due to the morpholine moiety attached, while compound $\mathbf{4 b}$ and $\mathbf{4 d}$ did not show any significant antibacterial activity. All the compounds 4(a-d) did not exhibit antifungal activity.

In vitro $\alpha$-Glucosidase inhibitory activity

All the eight Mannich bases were evaluated for $\alpha$ glucosidase inhibitory potential using acarbose as standard reference. The results were obtained in the form of $\%$ inhibition and $\mathrm{IC}_{50}$ were calculated graphically shown in Table II.

Table II

$\alpha$-Glucosidase inhibitory activity of Mannich bases 3(a-d) \& 4(a-d)

\begin{tabular}{|c|c|c|c|}
\hline Compound & Concentrations $(\mu \mathrm{g} / \mathrm{mL})$ & $\%$ of Inhibition & IC $_{50}$ value $(\mu \mathrm{g} / \mathrm{mL})$ \\
\hline \multirow{3}{*}{ 3a } & 60 & 30 & \multirow{3}{*}{$78.27 \pm 0.51$} \\
\hline & 80 & 42 & \\
\hline & 100 & 62 & \\
\hline \multirow{3}{*}{$3 \mathbf{b}$} & 60 & 40 & \multirow{3}{*}{$75.5 \pm 0.44$} \\
\hline & 80 & 47 & \\
\hline & 100 & 57 & \\
\hline \multirow{3}{*}{$3 c$} & 60 & 55 & \multirow{3}{*}{$53.58 \pm 0.22$} \\
\hline & 80 & 76 & \\
\hline & 100 & 85 & \\
\hline \multirow{3}{*}{ 3d } & 60 & 40 & \multirow{3}{*}{$68.87 \pm 0.11$} \\
\hline & 80 & 57 & \\
\hline & 100 & 66 & \\
\hline \multirow{3}{*}{$4 \mathbf{a}$} & 60 & 45 & \multirow{3}{*}{$60.39 \pm 0.43$} \\
\hline & 80 & 60 & \\
\hline & 100 & 67 & \\
\hline \multirow{3}{*}{$4 b$} & 60 & 55 & \multirow{3}{*}{$53.40 \pm 0.59$} \\
\hline & 80 & 62 & \\
\hline & 100 & 77 & \\
\hline \multirow{3}{*}{$4 c$} & 60 & 63 & \multirow{3}{*}{$43.05 \pm 0.11$} \\
\hline & 80 & 70 & \\
\hline & 100 & 82 & \\
\hline \multirow{3}{*}{$4 d$} & 60 & 43 & \multirow{3}{*}{$71.12 \pm 0.59$} \\
\hline & 80 & 52 & \\
\hline & 100 & 61 & \\
\hline \multirow{3}{*}{$\begin{array}{l}\text { Standard } \\
\text { drug } \\
\text { acarbose }\end{array}$} & 60 & 77 & \multirow{3}{*}{$27.10 \pm 0.51$} \\
\hline & 80 & 83 & \\
\hline & 100 & 94 & \\
\hline
\end{tabular}


FARMACIA, 2018, Vol. 66, 4

All the compounds demonstrated $\alpha$-glucosidase inhibitory activity with $\mathrm{IC}_{50}$ values ranging from $78.27 \pm 0.51 \mu \mathrm{g} / \mathrm{mL}$ to $43.05 \pm 0.11 \mu \mathrm{g} / \mathrm{mL}$. In this study compound $\mathbf{3 c}$ and $\mathbf{4 c}$ showed the highest inhibitory activity with $85 \%$ and $82 \%$ inhibition as compared with the standard drug acarbose $94 \%$ at concentration of $100 \mu \mathrm{g} / \mathrm{mL}$. The $\mathrm{IC}_{50}$ values of $\mathbf{3 c}$ was $53.58 \pm 0.22 \mu \mathrm{g} / \mathrm{mL}$ and that of $4 \mathbf{c}$ was $43.05 \pm$ $0.11 \mu \mathrm{g} / \mathrm{mL}$ which are closer to the $\mathrm{IC}_{50}$ of acarbose i.e. $27.10 \pm 0.51 \mu \mathrm{g} / \mathrm{mL}$. The higher activity can be attributed to the presence of morpholine moiety in both the Mannich base derivatives. The study also indicated that Mannich bases of 5-(2-hydroxyphenyl)1,3,4-oxadiazole-2(3H)-thione $(\mathbf{2 b})$ had lower $\mathrm{IC}_{50}$ as compared to corresponding Mannich bases of 5-(2aminoyphenyl)-1,3,4-oxadiazole-2(3H)-thione (2a). The $\mathrm{IC}_{50}$ values were calculated graphically by plotting log inhibitor concentration versus percent inhibition. So the study suggests that these Mannich bases can be taken as lead molecules for further improvements to act as effective $\alpha$-Glucosidase inhibitors.

\section{Isolation of binding sites}

Potential isolated binding sites of the target crystal structure of alpha-glucosidase (PDB ID: 3WY1) were analysed by Molecular Operating Environment (MOE) software. The possible binding sites of ligand molecules at alpha-glucosidase (PDB ID: 3WY1) protein are: ALA338, GLY342, ASP346, PRO347, HIS348, ALA349, TYR350, PRO351, LYS352, VAL353, LEU373, GLY374, LEU375, PRO376, ARG429, GLU432, LEU433, ALA434, ARG437, GLN438, ASP441, PRO442, ASN443, THR445, THR448, GLY496, ALA514, HIS515, PHE516, THR517, PRO528, ALA529, TYR530, GLN53 (Figure 2). Protein geometric arrangement of amino acid residues in allowed and disallowed regions indicate the quality of target proteins.

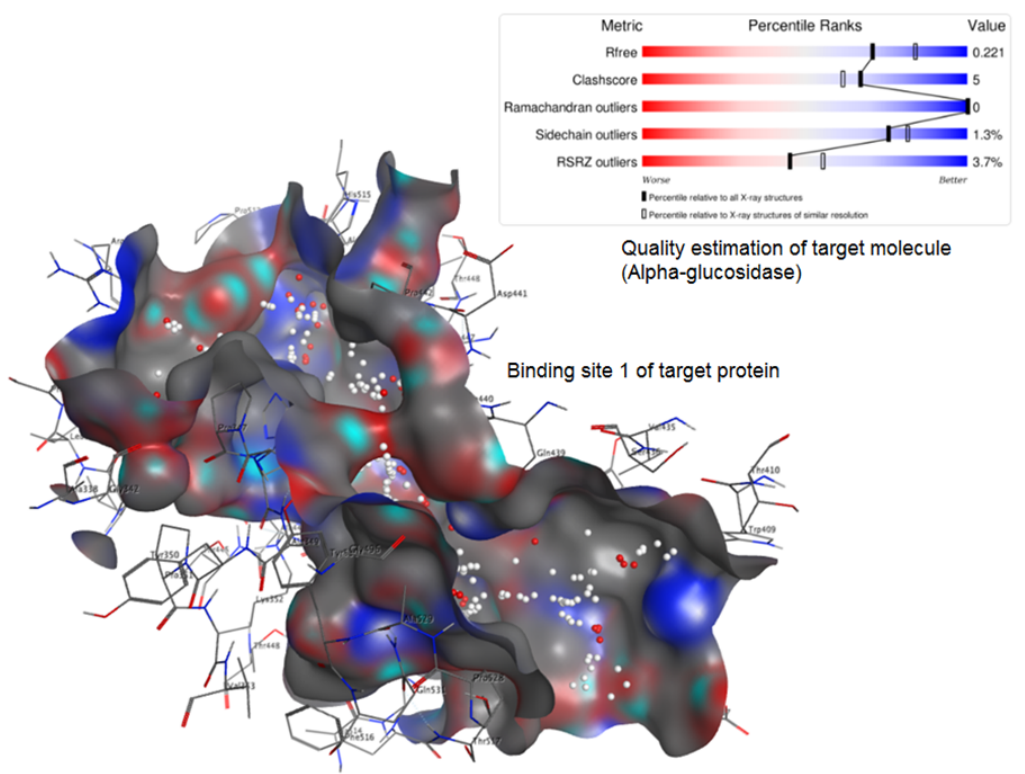

Figure 2.

Binding pocket of target protein $\alpha$-glucosidase (PDB ID: $3 \mathrm{WY} 1$ )

\section{Docking analysis}

Ligand binds to proteins to form stable complexes when molecular docking accounts for preferred ligand orientation. Minimum binding energy and scoring function determines the strength of this association i.e., ligand and protein. Computational ligand-target binding approach was employed in analysing these complexes of crystal structure of alpha-glucosidase (PDB ID: 3WY1) (target) with selected 8-compounds in order to interpret structural basis of target protein specificity. The interaction energy of compounds with target enzyme is assigned, "grid point". Finally, these ligands were docked with the potential active sites of target molecules. By the end of this process with numerous runs, binding energies were evaluated. Briefly, simulation, interaction energy of ligands and protein was measured utilizing atomic affinity potentials calculated on a grid at each step. Some of the parameters were set as default. Docking results were clustered and lowest binding energy cluster was considered as representative binding state. The minimum binding energies showed that target protein was docked successfully with ligand molecules (Table III).

All compounds were reviewed in detail to retrieve their binding interaction information that can be highly decisive for inhibition of targets. Binding interaction diagrams were obtained using MOE ligand interaction analysis feature (Figure 3). It is clear from the interaction analysis diagram that all the target molecules interact with the enzyme mostly through hydrophobic and hydrogen bonding interactions. 
Energy values obtained during docking analysis of series of compounds as ligand molecules against crystal structure of alpha-glucosidase (PDB ID: 3WY1) protein as target molecules

\begin{tabular}{|c|c|c|c|c|c|c|c|c|c|c|}
\hline S. No. & mol & rseq & mseq & $\mathbf{S}$ & rmsd_refine & E_conf & E_place & E_score1 & E_refine & E_score2 \\
\hline 1 & $\begin{array}{l}\text { 3a-glucosidase } \\
\text { interaction }\end{array}$ & 1 & 1 & -5.9173 & 1.4683 & 66.4669 & -52.8522 & -9.3077 & -15.738 & -5.9173 \\
\hline 2 & $\begin{array}{l}\text { 3b-glucosidase } \\
\text { interaction }\end{array}$ & 1 & 1 & -6.0419 & 3.0419 & 59.5528 & -68.9987 & -11.336 & -21.3354 & -6.0419 \\
\hline 3 & $\begin{array}{l}\text { 3c-glucosidase } \\
\text { interaction }\end{array}$ & 1 & 1 & -5.4736 & 2.4891 & 98.2656 & -78.1677 & -11.3329 & -15.8064 & -5.4736 \\
\hline 4 & $\begin{array}{l}\text { 3d-glucosidase } \\
\text { interaction }\end{array}$ & 1 & 1 & -5.879 & 1.4268 & 51.8011 & -57.9806 & -9.2159 & -11.1599 & -5.879 \\
\hline 5 & $\begin{array}{l}\text { 4a-glucosidase } \\
\text { interaction }\end{array}$ & 1 & 1 & -5.8469 & 2.7786 & -21.9165 & -57.922 & -9.5616 & -13.8401 & -5.8469 \\
\hline 6 & $\begin{array}{l}\text { 4b-glucosidase } \\
\text { interaction }\end{array}$ & 1 & 1 & -6.0083 & 1.9654 & -21.631 & -79.8785 & -12.9208 & -13.5468 & -6.0083 \\
\hline 7 & $\begin{array}{l}\text { 4c-glucosidase } \\
\text { interaction }\end{array}$ & 1 & 1 & -5.4795 & 3.0108 & 83.6733 & -80.1395 & -11.179 & -20.0708 & -5.4795 \\
\hline 8 & $\begin{array}{l}\text { 4d-glucosidase } \\
\text { interaction }\end{array}$ & 1 & 1 & -5.3836 & 2.4939 & 42.1492 & -51.5655 & -10.984 & -10.1349 & -5.3836 \\
\hline
\end{tabular}

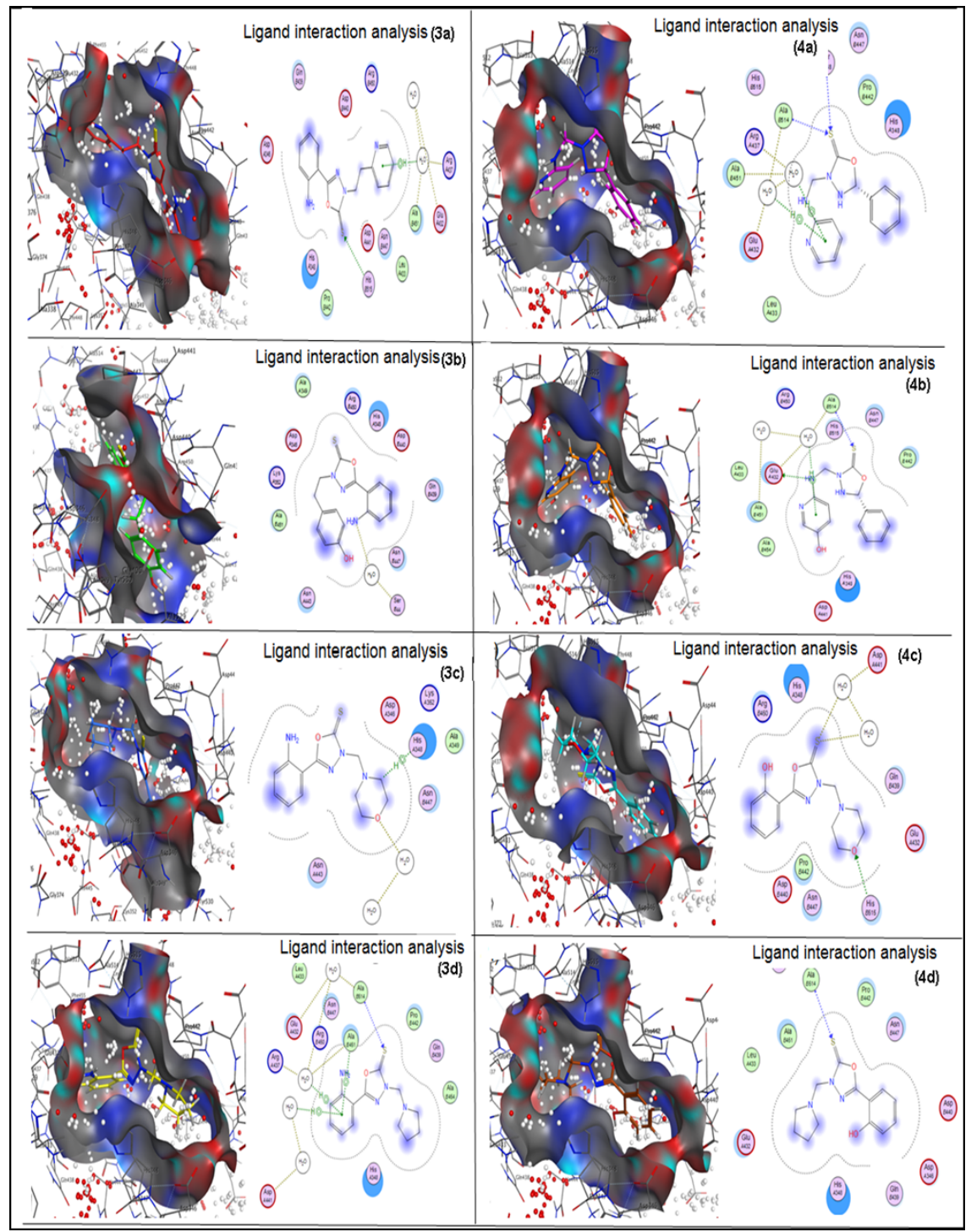

Figure 3.

Ligand-protein interaction analysis of synthesized Mannich bases 3(a-d) and 4(a-d) 
FARMACIA, 2018, Vol. 66, 4

\section{Conclusions}

In the current study eight new Mannich bases of 1,3,4oxadiazole-2-thiol were synthesized and evaluated for their antimicrobial and $\alpha$-glucosidase inhibitory potential. All the synthesized Mannich bases 3(a-d) and 4(a-d) did not show significant antimicrobial activity, however all compounds showed reasonable $\alpha$-glucosidase inhibitory activity. Highest activity was exhibited by $\mathbf{3 c}$ and $\mathbf{4 c}$ containing morpholine moiety, having $\mathrm{IC}_{50}$ of $53.58 \pm 0.22$ and $43.05 \pm 0.11$ respectively. These compounds inhibited $\alpha$ glucosidase activity by $85 \%$ and $82 \%$ at concentration of $100 \mu \mathrm{M}$.

Molecular docking results also supported the interactions of all Mannich bases with $\alpha$-glucosidase, especially compounds $\mathbf{3 b}, \mathbf{3 c}, \mathbf{4 b}$ and $\mathbf{4 c}$ showed good interaction network. Based on the results, these morpholine containing Mannich bases of mercapto oxadiazole can be considered as lead in search of new $\alpha$-glucosidase inhibitors.

\section{References}

1. Aanandhi MV, Verma AK, Sujatha R, Raj RK, Synthesis and characterization of novel Mannich bases of benzimidazole derivatives for antibacterial and antifungal activity. Int $J$ Pharm Pharmaceut Sci., 2013; 5(2):295-297.

2. Aboraia AS, Abdel-Rehman HM, Mahfouz NM, El-Gendy MA, Novel 5-(2-hydroxyphenyl)-3substituted-2,3-dihydro-1,3,4-oxadiazole-2-thione derivatives: Promising anticancer agents. Bioorg Med Chem., 2016; 14: 1236-1246.

3. Ali MA, Shaharyar M, Oxadiazole Mannich bases: Synthesis and antimycobacterial activity. Bioorg Med Chem Lett., 2007; 17: 3314-3316.

4. Almasirad A, Vousooghi N, Tabatabai SA, Shafiee A, Synthesis, Anticonvulsant and Muscle Relaxant Activities of Substituted 1,3,4-oxadiazole, 1,3,4thiadiazole and 1,2,4-triazole. Acta Chim Slov., 2007; 54: 317-324.

5. Bala S, Sharma N, Kajal A, Kamboj S, Design, synthesis, characterization and computational studies on benzamide substituted Mannich bases as novel, potential antibacterial agents. Sci World J., 2014; 2014: 1-9.

6. Balouiri M, Sadiki M, Ibnsouda SK, Methods for in vitro evaluating antimicrobial activity: A review. $J$ Pharm Anal., 2016; 6(2): 71-79.

7. Chen CJ, Song BA, Yang S, Xu GF, Bhadury PS, Jin LH, Hu DY, Li QZ, Liu F, Xue W, Lu P, Chen $Z$, Synthesis and antifungal activities of 5-(3,4,5trimethoxyphenyl)-2-sulfonyl-1,3,4-thiadiazole and 5-(3,4,5-trimethoxyphenyl)-2-sulfonyl-1,3,4-oxadiazole derivatives. Bioorg Med Chem., 2007; 15(12): 3981-3989.

8. Cnop M, Welsh N, Jonas JC, Jörns A, Lenzen S, Eizirik DL, Mechanisms of pancreatic beta-cell death in type 1 and type 2 diabetes: many differences, few similarities. Diabetes, 2005; 54: S97.
9. Dilmaghani KA, Pur FN, Pour MM, Nejad JM, Novel Oxadiazole Thioglycosides as Potential Anti-Acinetobacter Agents. Iran J Pharm Res., 2016; 15(4): 777-778.

10. Dimmock JR, Kumar P, Anticancer and cytotoxic properties of Mannich bases. Curr Med Chem., 1997; 4: 1-22.

11. El-Sayed WA, El-Essawy FA, Ali OM, Nasr BS, Abdalla MM, Abdel-Rahman AAH, Anti-HIV activity of new substituted 1, 3, 4-oxadiazole derivatives and their acyclic nucleoside analogues. Zeitschrift für Naturforschung C, 2009; 64(11): 773-778.

12. Expert Committee on the Diagnosis and Classification of Diabetes Mellitus, Report of the Expert Committee on the Diagnosis and Classification of Diabetes Mellitus. Diabetes Care, 1997; 20: 1183.

13. Idhayadhulla A, Kumar RSA, Nasser AJA, Manilal A, Synthesis and antimicrobial activity of some new Mannich base derivatives. J Chem Pharm Res., 2011; 3(4): 904-911.

14. Kadi AA, El-Brollosy NR, Al-Deeb OA, Habib EE, Ibrahim TM, El-Emam AA, Synthesis, antimicrobial, and anti-inflammatory activities of novel 2-(1adamantyl)-5-substituted-1,3,4-oxadiazoles and 2-(1adamantylamino)-5-substituted-1,3,4-thiadiazoles. Eur J Med Chem., 2007; 42(2): 235-242.

15. Kanthiah S, Kalusalingam A, Velayutham R, Vimala AT, 5-(2-aminophenyl)-1, 3, 4-oxadiazole-2 (3H)thione derivatives: Synthesis, characterization and antimicrobial evaluation. Int J Pharm Sci., 2011; 6(1): 64-67.

16. Keyvan P, Leila B, Zahra R, Manuchehr S, Kamiar $\mathrm{Z}$, In vitro activity of six antifungal drugs against clinically important dermatophytes. Jundi J Microbiol., 2009; 4: 158-163.

17. Khan KM, Fatima N, Rasheed M, Jalil S, Ambreen N, Perveen S, Choudhary MI, 1,3,4-Oxadiazole$2(3 \mathrm{H})$-thione and its analogues: A new class of non-competitive nucleotide pyrophosphatases/ phosphodiesterases 1 inhibitors. Bioorg Med Chem., 2009; 17: 7816-7822.

18. Mandour A, Fawzy N, El-Shihi T, El-Bazza Z, Synthesis, antimicrobial and antiaflatoxigenic activities of some Benzofuran containing 1,2,4-Triazole, 1,3,4-Thiadiazole and oxadiazole derivatives. Pak J Sci Ind Res., 1995; 38: 402.

19. Omar MT, Synthesis of new xanthenone derivatives of expected antibilharzial activity. Arch Pharm Res., 1997; 20(6): 602-609.

20. Padmavathi V, Sudhakar G, Reddy A, Padmaja P, Ali $\mathrm{S}$, Synthesis, antimicrobial and cytotoxic activities of 1,3,4-oxadiazoles, 1,3,4-thiadiazoles and 1,2,4triazoles. Eur J Med Chem., 2009; 44(5): 2106.

21. Pandeya SN, Sriram D, Nath G, Clercq ED, Synthesis, antibacterial, antifungal and anti-HIV activities of norfloxacin Mannich bases. Eur J Med Chem., 2000; 35(2): 249-255.

22. Playford RJ, Pither C, Gao R, Middleton SJ, Use of the a-glucosidase inhibitor acarbose in patients with 'Middleton syndrome': normal gastric anatomy but with accelerated gastric emptying causing postprandial reactive hypoglycemia and diarrhea. Can J Gastroenter., 2013; 27: 403-404. 
23. Ragenovic KC, Dimova V, Kakurinov V, Molnar DG, Buzarovska A, Synthesis, Antibacterial and Antifungal Activity of 4-Substituted-5-Aryl-1,2,4Triazoles. Molecules, 2001; 6: 815-824.

24. Rahul VP, Kumari P, Chikhalia K, New Quinolinyl1,3,4-Oxadiazoles: Synthesis, In Vitro Antibacterial, Antifungal and Antituberculosis Studies. Med Chem., 2013; 9: 596-607.

25. Selvakumar K, Rubu JJ, Rajamanickam V, Vignesh M, Parkavi V, Mohamed JM, Beyatricks KJ, Synthesis, Characterization and analgesic activity of 1,3,4Oxadiazole derivatives. Int $J$ Pharm \& Ind Res., 2012; 2(1): 6-9.

26. Shahzad SA, Yar M, Bajda M, Jadoon B, Khan ZA, Naqvi SAR, Shaikh AJ, Hayat K, Mahmood A, Mahmood N, Filipek S, Synthesis and biological evaluation of novel oxadiazole derivatives: a new class of thymidine phosphorylase inhibitors as potential anti-tumor agents. Bioorg Med Chem., 2014; 22(3): 1008-1015.

27. Shen S, Sun X, Liu Y, Chen B, Jin R, Ma H, Synthesis and Biological Activity of Some Novel Aryl-
Substituted 1,3,4-Oxadiazole Mannich Bases Containing Pyrimidine Rings. J Hetro Chem., 2015; 52: 12961301.

28. Siddiqui SM, Salahuddin A, Azam A, Mannich base derivatives of 1,3,4-oxadiazole: synthesis and screening against Entamoeba histolytica. Med Chem Res., 2012; 22: 1313-1319.

29. Sies H., Oxidative stress: oxidants and antioxidants. Exp Physio., 1997; 82: 291-295.

30. Subramaniapillai SG, Mannich reaction: a versatile and convenient approach to bioactive skeletons. $J$ Chem Sci., 2013; 125: 467-482.

31. Taha M, Ismail NH, Imran S, Wadood A, Rahim F, Ali M, Rehman AU, Novel quinoline derivatives as potent in vitro $\alpha$-glucosidase inhibitors: in silico studies and SAR predictions. Med Chem., 2015; 6(10): 1826-1836.

32. Vogel AI, A text book of practical organic chemistry, $5^{\text {th }}$ ed/rev by Brian S. Furness et al., John Wiley and Sons, Inc. New York, 1989; 0434. 